\title{
Supernatants from culture of type I collagen-stimulated PBMC from patients with cutaneous systemic sclerosis versus localized scleroderma demonstrate suppression of MMP-1 by fibroblasts
}

\author{
Monica Brown • Arnold E. Postlethwaite • \\ Linda K. Myers • Karen A. Hasty \\ Received: 19 October 2011 /Revised: 2 December 2011 / Accepted: 7 February 2012 / Published online: 25 February 2012 \\ (C) The Author(s) 2012. This article is published with open access at Springerlink.com
}

\begin{abstract}
Systemic sclerosis ( $\mathrm{SSc}$ ) is a chronic fibrosing disease characterized by vasculopathy, autoimmunity, and an accumulation of collagen in tissues. Numerous studies have shown that compared to healthy or diseased controls, the peripheral blood mononuclear cells (PBMC) from patients with SSc produce a variety of cytokines or proliferate when cultured with solubilized type I collagen (CI) or constituent $\alpha 1$ (II) and $\alpha 2$ (I) polypeptide chains. The purpose of this study was to determine whether PBMC isolated from patients with SSc and cultured in vitro with soluble CI elaborated soluble mediators that inhibit the production of collagenase (i.e., matrix metalloproteinase, MMP-1) by fibroblasts. Supernatants of CIstimulated PBMC from juvenile and adult diffuse cutaneous
\end{abstract}

Drs. Brown and Postlethwaite contributed equally to the paper.

M. Brown $(\bowtie) \cdot$ L. K. Myers

Le Bonheur Children's Medical Center,

Department of Pediatrics (Division of Clinical Immunology,

Section of Rheumatology),

University of Tennessee Health Science Center,

956 Court Avenue, Suite G326,

Memphis, TN 38163, USA

e-mail: mbrown67@uthsc.edu

A. E. Postlethwaite $\cdot$ L. K. Myers $\cdot$ K. A. Hasty Department of Medicine (Division of Connective Tissue Diseases), University of Tennessee Health Science Center, Memphis, TN 38163, USA

A. E. Postlethwaite $\cdot$ L. K. Myers - K. A. Hasty Department of Veterans Affairs Medical Center, Memphis, TN 38104, USA

A. E. Postlethwaite $\cdot$ K. A. Hasty

Department of Orthopedic Surgery,

University of Tennessee Health Science Center,

Memphis, TN 38163, USA
(dc)SSc patients significantly reduced MMP-1 production by SSc dermal fibroblasts, while supernatants of CI-stimulated PBMC from patients with localized scleroderma (LS) did not. CI-stimulated PBMC culture supernatants from patients with $\mathrm{dcSSc}$ in contrast to patients with LS exhibited increased levels of platelet-derived growth factor (PDGF)-AA, PDGFBB, TNF- $\alpha$, IL-13, and EGF. Prolonged culture of SSc dermal fibroblasts with recombinant PDGF-BB or IL-13 inhibited the induction of MMP-1 in response to subsequent TNF- $\alpha$ stimulation. These data suggest that therapies aimed at reducing these cytokines may decrease collagen accumulation in SSc, preventing the development of chronic fibrosis.

Keywords IL-13 · Diffuse - Localized scleroderma · MMP-1 . PBMC $\cdot$ PDGF-BB $\cdot$ Scleroderma

\section{Introduction}

Scleroderma "fibrosis of the skin" spectrum disorders include localized scleroderma (LS) variants and systemic sclerosis (SSc) the latter of which is further divided into diffuse cutaneous (dc)SSc, limited cutaneous (lc) SSc, and SSc sine scleroderma. The forms of SSc are differentiated primarily on the basis of the pattern of skin involvement, autoantibody association, and characteristics of internal organ involvement [1]. The main clinical differences between LS and SSc are the absence in the former of sclerodactyly, Raynaud's phenomenon, absence of widespread vasculopathy, nail fold capillary changes, and internal organ fibrosis. Five subtypes of LS or morphea are recognized by Peterson et al. and include "plaque," "generalized," "bullous," "linear," and "deep" [2]. The morphea classification of Laxer and Zulian [3] adds additional 
subgroups and recognizes "mixed variant morphea" which includes a combination of two or more subtypes in the same patient. Immunologic tests show that although autoantibodies are highly prevalent in both LS and SSc, they differ in prevalence and antigen specificity in these major divisions of scleroderma spectrum disease. For example, antinuclear antibodies are present in only $20 \%$ to $80 \%$ of patients with LS and in $>90 \%$ of patients with SSc $[4,5]$. Moreover, antitopoisomerase II alpha antibody is present in up to $85 \%$ of patients with LS and in 14\% of patients with SSc [4].

In this present study, we show profound differences in the immune responses of patients with dcSSC compared to patients with LS. Peripheral blood mononuclear cells (PBMC) from patients with dcSSc, but not patients with LS, cultured in vitro with soluble CI or $\alpha 1$ (I) generate cytokine/growth factor-rich supernatants. These supernatants induce a reduction in matrix metalloproteinase (MMP)-1 production, when added to cultures of SSc dermal fibroblasts, but not to normal donor dermal fibroblast lines. We hypothesize that $\mathrm{T}$ cells from patients with dcSSc are activated by CI to produce cytokines that act on fibroblasts to reduce synthesis of MMP-1. A decrease in the skin fibroblast's ability to synthesize MMP-1 could shift the balance of collagen metabolism toward collagen excess resulting in fibrotic lesions.

In these studies, PBMC from patients with dcSSc or LS were cultured with or without $\mathrm{CI}$, and the harvested supernatants were added to cultures of SSc fibroblasts to evaluate the effects on MMP-1 production. We found that long-term exposure to supernatants that had been generated by culturing CI-stimulated PBMC from adult or pediatric patients with dcSSc caused a significant decrease in the production of MMP-1 by SSc fibroblasts. In contrast, long-term exposure of SSc fibroblasts to supernatants from CI-stimulated PBMC from patients with LS did not induce suppression of MMP-1 production by tumor necrosis factor alpha (TNF- $\alpha$ )stimulated dcSSc fibroblasts. These data suggest that therapies aimed at reducing the suppression of MMP may decrease collagen accumulation in skin and vital organs of patients with SSC, preventing the development of chronic fibrosis. Moreover, the results of this study may help elucidate the pathogenesis of impaired MMP-1 production by fibroblasts in SSc.

\section{Results}

Inhibition of MMP-1 production by dcSSc dermal fibroblasts chronically exposed in vitro to pooled culture supernatants from CI-stimulated PBMC from patients with dcSSc To determine whether cytokine/growth factors present in supernatants from culture of CI-stimulated SSc PBMC would effect a change in MMP-1 production by dermal fibroblasts, we obtained PBMC from ten adult patients with dcSSc and stimulated them in vitro with CI or medium alone for 6 days. The harvested supernatants were pooled and added at $30 \%$ $v / v$ to a culture of dermal fibroblasts from normal donors and from involved skin of patients with dcSSc for 14 days as described in the Patients and methods section, and MMP-1 production in response to TNF- $\alpha$ stimulation was assessed.

As shown in Table 1, culture of dcSSc fibroblasts for 14 days with supernatants from CI-stimulated adult dcSSc PBMC resulted in significant reduction in MMP-1 protein produced in response to TNF- $\alpha$ stimulation. Compared to normal fibroblasts, production of MMP-1 was also reduced in the dcSSc fibroblast cultures not stimulated with TNF- $\alpha$ but cultured for 14 days with CI-stimulated PBMC from patients with dcSSc. In contrast, fibroblasts from normal donors cultured for 2 weeks with the same pooled supernatants from CI-stimulated dcSSc PBMC and stimulated with TNF- $\alpha$ showed an actual enhancement of MMP-1 (Table 1).

Since the spectrum of scleroderma can range from very minimal involvement (LS) to extensive fibrosis $(\mathrm{dcSSc})$, it was of interest to compare the effect of supernatants from the culture of PBMC from patients with LS to supernatants from the culture of PBMC from patients with dcSSc on MMP-1 production by SSc fibroblasts. In order to determine the variability among individual patients, we cultured PBMC with and without CI for 6 days and studied the effect of each individual donor PBMC supernatant on a single $\mathrm{dcSSC}$ dermal fibroblast line (SSc008) that became responsive (after subpassage 4) to MMP-1 upregulation by TNF- $\alpha$.

PBMC from four dcSSc patients (one juvenile and three adults) and five juvenile patients with LS were cultured with or without native CI for 6 days. The harvested supernatants from the culture of PBMC from each of these patients were added at $30 \% v / v$ to cultures of dermal fibroblasts ( $\mathrm{SSc} 008)$ for 21 days with fresh media and PBMC supernatant added every 3 days. After 21 days, TNF- $\alpha$ was added to the cultures of SSc008 fibroblasts, to stimulate production of MMP-1. As illustrated in Fig. 1, the SSc 008 fibroblast line produced significantly less MMP-1 after being cultured for 21 days with CI-stimulated supernatants from dcSSc patients compared to fibroblasts cultured with CI-stimulated supernatants from LS patients.

Cytokine profile for CI-stimulated PBMC supernatants from juvenile and adult dcSSc The inhibition of MMP-1 described above is very likely induced by cytokines or growth factors secreted by peripheral blood cells. In order to determine the cytokine profile of supernatants from culture of CI-stimulated dcSSc PBMC, we pooled CI-stimulated PBMC culture supernatants from ten adult patients with dcSSc and incubated these supernatants on RayBioplex membrane arrays to test for presence of individual cytokines/growth factors. The pooled supernatants were tested on three different 
Table 1 Effect of supernatant from type I collagen-stimulated PBMC (from patients with deSSc) on MMP-1 production by deSSc and normal fibroblasts

Additions for $48 \mathrm{~h}$ dcSSc fibroblast lines Mean $\pm \mathrm{SE}$ Normal donor fibroblast Mean $\pm \mathrm{SE} P$ value lines

\begin{tabular}{|c|c|c|c|c|c|c|c|c|c|c|c|c|}
\hline \multirow[t]{3}{*}{ Fibroblasts cultured with medium } & & 004 & 008 & 1685 & 1822 & & 1 & 3 & 2603 & 6858 & & \multirow{3}{*}{$\mathrm{NS}^{\mathrm{b}}$} \\
\hline & $\mathrm{PBS}^{\mathrm{a}}$ & 4 & 18 & 69 & 38 & $32 \pm 14$ & 5 & 15 & 60 & 29 & $27 \pm 12$ & \\
\hline & TNF- $\alpha(5 \mathrm{ng} / \mathrm{ml})$ & 100 & 100 & 100 & 100 & 100 & 100 & 100 & 100 & 100 & 100 & \\
\hline \multirow{2}{*}{$\begin{array}{l}\text { Fibroblasts cultured with } 30 \% v / v \\
\text { CI-stimulated dcSSc PBMC supernatant }\end{array}$} & $\mathrm{PBS}^{\mathrm{a}}$ & 2 & 15 & 27 & 23 & $17 \pm 5$ & 125 & 69 & 92 & 22 & $77 \pm 22$ & $0.036^{\mathrm{b}}$ \\
\hline & TNF- $\alpha(5 \mathrm{ng} / \mathrm{ml})^{\mathrm{a}}$ & 54 & 60 & 64 & 34 & $53 \pm 7$ & 578 & 413 & 210 & 106 & $327 \pm 105$ & $0.041^{\mathrm{c}}$ \\
\hline
\end{tabular}

MMP1 was measured by Western blot using pooled supernatants of CI-stimulated PBMC from ten dcSSc patients. The pooled PBMC supernatants were added at $30 \% v / v$ to cultures of normal and dsSSc fibroblasts for 14 days as described in the "Patients and methods" section. Normal and dcSSc fibroblasts cultured with pooled $(n=10)$ CI-stimulated dcSSc PBMC supernatants (bottom panel) were compared to the same cells cultured with the medium alone (top panel). The MMP-1 response to TNF- $\alpha$ in the medium alone was arbitrarily set at $100 \%$

${ }^{\text {a }}$ MMP-1 calculated as a percent of that seen with TNF- $\alpha$ stimulation in fibroblasts cultured with the medium alone

${ }^{b}$ MMP-1 produced by SSc fibroblasts compared to normal dermal fibroblast cell lines following treatment with PBS

${ }^{\mathrm{c}}$ MMP-1 produced by SSc fibroblasts compared to normal dermal fibroblast cell lines following treatment with TNF- $\alpha$

cytokine or growth factor arrays and were found to be abundant in platelet-derived growth factor (PDGF)-AA, PDGF$\mathrm{BB}, \mathrm{PDGF}-\mathrm{AB}$, epidermal growth factor (EGF), insulin-like growth factor binding protein 2 (IGFBP-2), hepatocyte growth factor (HGF), macrophage colony stimulating factor receptor (MCSFR), and interleukin-13 (IL-13) when compared with the culture medium alone (Fig. 2). We further evaluated the cytokine profile of supernatants from CIstimulated PBMC from one pediatric dcSSC patient (Fig. 3, P003) and two pediatric LS patients (Fig. 3, P001, P002). We cultured PBMC with CI for 6 days from these patients, harvested the supernatant, and incubated these supernatants on RayBioplex membrane arrays as per manufacturer's instructions. As shown in Fig. 3, the cytokine profile of supernatants from CI-stimulated PBMC from the juvenile dcSSc patient revealed an increase in PDGF-AA, PDGF-BB, IL-13, TNF- $\alpha$, and EGF when compared to the supernatant from CIstimulated PBMC from patients with LS. Although the numbers are small, the PBMC from the dcSSC patient clearly secreted greater amounts of PDGF-AA, PDGF-BB, IL-13, TNF- $\alpha$, and EGF when compared to supernatants collected from the culture of CI-stimulated PBMC from LS patients. These results compare favorably to the cytokine profile in supernatants from the culture of CI-stimulated PBMC from adult patients with dcSSc (Figs. 2 and 3).

Effects of long-term incubation of SSc dermal fibroblast with profibrotic cytokines In a separate experiment, PDGF-AA, PDGF-BB, IL-13, and EGF were added for 14 days individually at varying doses to cultures of SSc fibroblasts to assess their effect on MMP-1 production induced by TNF- $\alpha$. Although multiple concentrations were tested, the data shown in Fig. 4 illustrate the most effective dose for each cytokine. Long-term exposure of SSc 008 fibroblasts to PDGF-BB and IL-13 significantly inhibited
MMP-1 production when the fibroblasts were subsequently stimulated with TNF- $\alpha$. These data suggest that each of these cytokines may be responsible for the inhibitory effect on fibroblast MMP-1 production induced by supernatants from CI-stimulated PBMC from patients with dcSSc. In contrast, long-term exposure of SSc 008 fibroblasts to PDGF-AA and EGF resulted in enhanced TNF- $\alpha$ stimulation of MMP-1 production (Fig. 4). Although EGF is known to upregulate TGF- $\beta$ via PI 3-kinase/Akt signaling pathway in dermal fibroblasts [6], the upregulation of TGF- $\beta$ by EGF in the present experiments would not impact MMP-1 production, as activation of latent TGF- $\beta$ does not occur spontaneously in culture, and active TGF- $\beta$ suppresses MMP-1 production by fibroblasts [7].

\section{Discussion}

Our results show that soluble mediators from PBMC from patients with dcSSc inhibit collagenase expression in cultured SSc dermal fibroblasts. Supernatants of soluble CI-stimulated PBMC from dcSSc patients exerted profound suppression of production of MMP-1 by SSc fibroblasts. Pooled supernatants from a group of adult dcSSc patients and individual supernatants from juvenile and adult dcSSc patients significantly reduced the expression of MMP-1 by SSc fibroblasts. Suppression of constitutive production as well as TNF- $\alpha$ stimulated production of MMP-1 by SSc fibroblasts was observed when these fibroblasts were chronically exposed to supernatants from CI-stimulated dcSSc PBMC. In contrast, prolonged culture of SSc fibroblasts with supernatants of CIstimulated PBMC from patients with LS did not induce the MMP-1 suppressive phenotype in SSc fibroblasts.

TNF- $\alpha$ inhibits collagen synthesis and increases synthesis of MMP-1 when added to cultures of normal human 


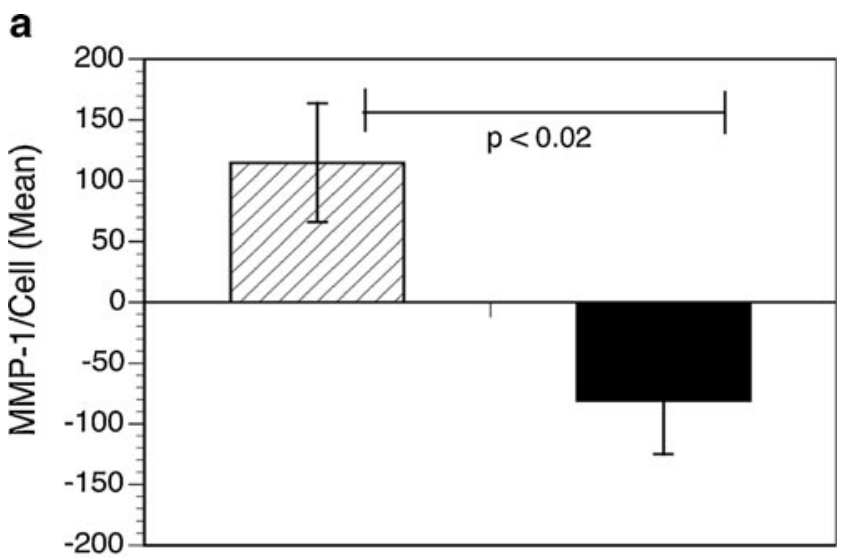

b

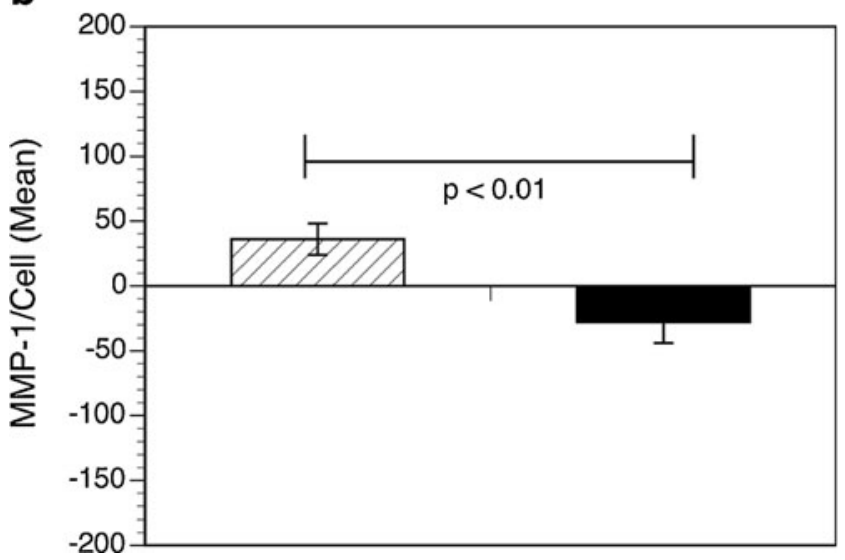

Fig. 1 The effect of juvenile and adult dcSSc and LS PBMC supernatants on MMP-1 production by SSc fibroblasts. PBMC isolated from patients with dcSSc $(n=4$; solid bars) and LS ( $n=5$; hatched bars) were stimulated with and without CI for 6 days, and the individual supernatants were tested for their effect on the MMP-1 production by $\mathrm{SSc}$ fibroblasts. Dermal fibroblasts from a patient with SSc (SSc008) were cultured for 21 days each with PBMC supernatants with (a) and without CI (b) after stimulation with TNF- $\alpha$ for $24 \mathrm{~h}$. The amount of MMP-1 accumulating in the culture supernatants is shown measured by Western blot calculated as a percentage of MMP-1 from fibroblasts that were not co-cultured with PBMC supernatant, but were stimulated with TNF- $\alpha$ (control). Results were confirmed by ELISA (not shown). Results are expressed as mean \pm SEM for MMP-1 content of the medium from the fibroblast cultures

fibroblasts [8]. Unstimulated PBMC from patients with lcor $\mathrm{dcSSc}$ in culture produce greater amounts of TNF- $\alpha$ than PBMC cultured from healthy volunteers, and the most TNF$\alpha$ was produced by unstimulated PBMC from patients with early (<3-year duration) dcSSc [9]. Higher amounts of TNF$\alpha$ are also expressed in the skin of patients with SSc [10]. Although TNF- $\alpha$ is abundantly produced in SSc patients, our data in this report suggest that cytokines produced by CI-activated PBMC from patients with deSSc may render fibroblasts insensitive to TNF- $\alpha$ upregulation of MMP-1 and thereby contribute to dermal fibrosis.

The pathogenesis of fibrosis in LS and SSc is still unresolved, but a role for autoimmunity in effecting fibrosis is suggested by early tissue infiltrates of mononuclear cells that have the capability of producing an array of cytokines and growth factors that are known to mediate fibrogenesis. The histologic features of the lesional skin of patients with LS and SSc are largely indistinguishable and are characterized in the early stages by perivascular infiltration of predominantly mature lymphocytes, $\mathrm{T}$ lymphoblasts, immature plasma cells, mature plasma cells, fibroblasts, fibrocytes, fibroblast-like cells, macrophages, undifferentiated mesenchymal cells, and monocytes [11]. Later, additional reports have described the cellular infiltrates more precisely. Roumm et al. analyzed the infiltrates in the lesional skin of patients with SSc using monoclonal antibodies to cell surface markers and found the mononuclear cell (MNC) infiltrates were composed predominately of activated $\mathrm{CD}^{+}$and $\mathrm{CD}^{+} \mathrm{T}$ cells in a ratio of 2.4 to 1 , and few $\mathrm{B}$ cells and monocytes were observed [12]. Importantly, in this larger cohort study of 115 patients with SSc, significant correlations were observed between the degree of MNC infiltration and both the degree and progression of skin thickening [12].

Separate studies have shown that when PBMC from patients with lc or dcSSc (in contrast to healthy volunteers) are cultured with soluble CI or constituent $\alpha 1$ (I) or $\alpha 2(\mathrm{I})$ chains, there is increased production of cytokines including IL-2, IL-6, IFN- $\gamma$, IL-10; increased production of monocyte chemoattractants, and increased T cell proliferation [13-18]. In addition, Warrington et al. labeled PBMC from patients with $\mathrm{lcSSc}$, dcSSc, and rheumatoid arthritis and healthy volunteers with carboxyfluorescein diacetate succinimidyl ester (CFSE) and cultured these CFSE-labeled PBMC for 14 days with and without soluble native bovine $\mathrm{CI}$ and $\beta 1$, two chains [dimers formed by $\alpha 1$ (I) and $\alpha 2(\mathrm{I})$ ] [19]. Analysis by flow cytometry showed a $\mathrm{T}$ cell proliferative response to these CI $\alpha$ chains occurring in $32 \%$ of SSc patients but only in $3.6 \%$ healthy volunteers ( 1 of 19 volunteers) and in $0 \%$ RA patients ( 0 of 9 ) [19]. The proliferating $\mathrm{T}$ cells expressed $\mathrm{CD} 4^{+}$, activated $\left(\mathrm{CD} 25^{+}\right)$memory $\left(\mathrm{CD} 45 \mathrm{RO}^{+}\right.$) phenotype [19]. The $\mathrm{T}$ cell lines generated by prolonged culture (20-30 days culture) of sorted CI-reactive T cells with CI, irradiated autologous PBMC, and IL-2 were analyzed after resting for 10 days and then stimulated for $72 \mathrm{~h}$ with plate-bound anti-CD3 and anti CD28. These T cell lines then produced abundant Th1 cytokines, IFN- $\gamma$, IL-2, TNF- $\alpha$, lower amounts of Th2 cytokines (IL-4, IL-6, and IL-10), and abundant chemotactic chemokine IL-8 [19].

While this study does not establish whether the diminished production of MMP-1 that we have observed is due to T cell autoimmunity to CI or due to a costimulatory effect of $\mathrm{CI}$ on activated $\mathrm{T}$ cells, we favor an antigenic role of $\mathrm{CI}$ in triggering production of cytokines. We have previously shown that CI-specific $\mathrm{T}$ cell clones can be grown from peripheral blood of SSc patients [19]. Moreover, the fact 


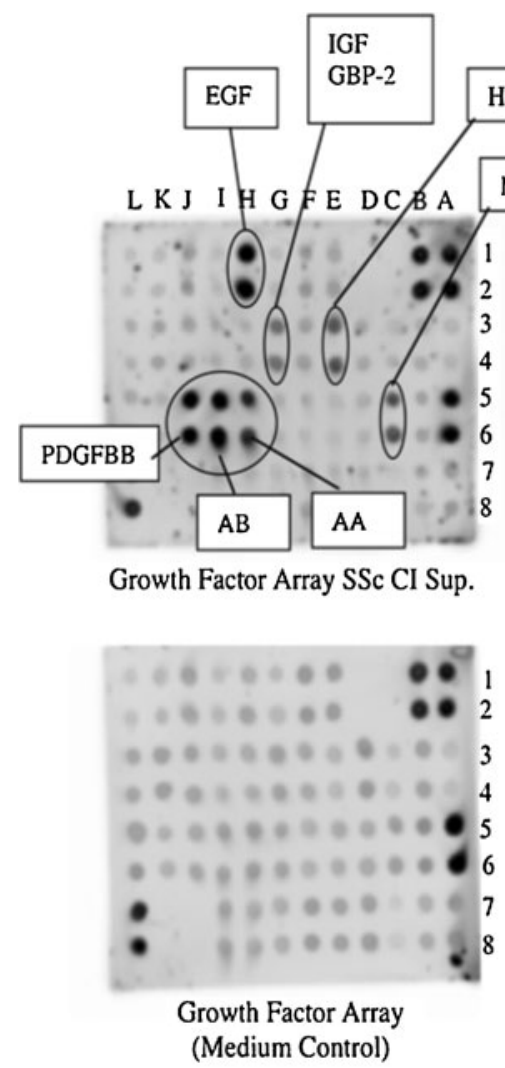

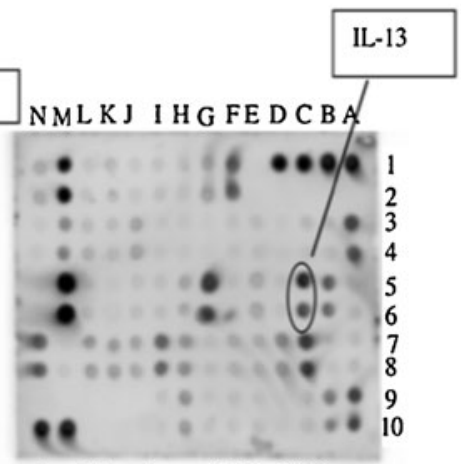

Cytokine Array vi SSc CI Sup.

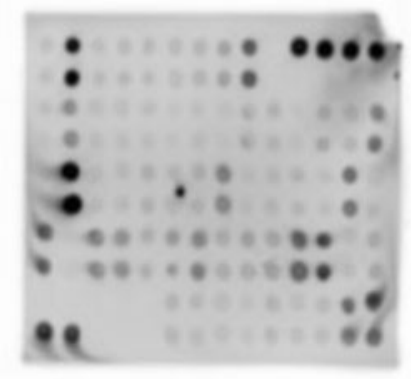

Cytokine Array vi

(Medium Control)
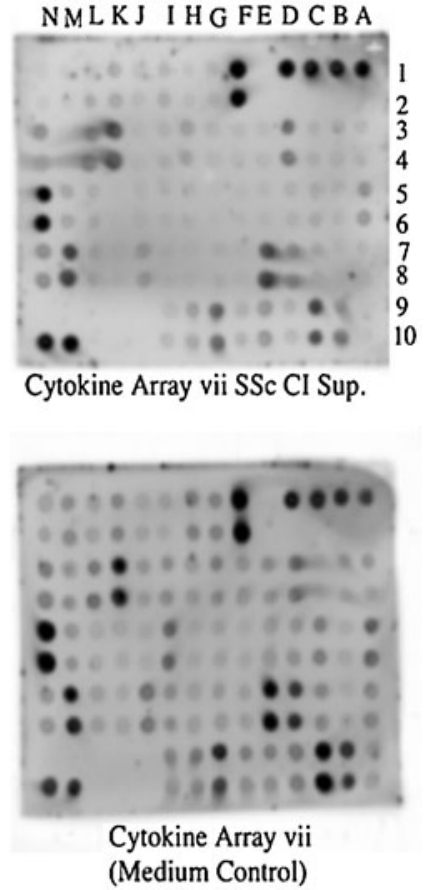

Fig. 2 RayBio Human Cytokine Antibody Array of pooled culture supernatants from PBMC from ten adult patients with dcSSc stimulated for 6 days with $10 \mu \mathrm{g} / \mathrm{ml}$ native bovine CI. After 6 days of incubation of PBMC plus $10 \mu \mathrm{g} / \mathrm{ml} \mathrm{CI}$, culture supernatants were pooled and analyzed, along with the culture medium alone (complete DMEM) as a control, in a RayBio Cytokine and Growth Factor Antibody Array assay. The assays used contained antibodies to 120

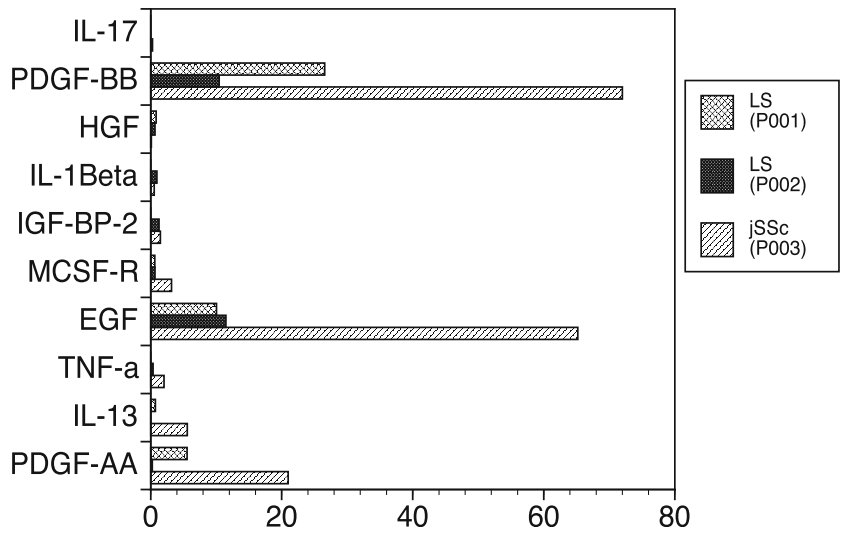

Fig. 3 Cytokine profile supernatants from juvenile dcSSc and LS PBMC stimulated with CI. PBMC from one patient with dcSSc (P003) and two patients with LS (P002 and P001) were cultured individually for 6 days with CI, and the supernatants were collected and analyzed to be tested using RayBio Cytokine and Growth Factor Antibody Array assay. The data points represent individual patients. The patient with dcSSC had increased PDGF-AA, PDGF-BB, IL-13, TNF- $\alpha$, and EGF as compared to PBMC from LS patients (P002 and $\mathrm{P} 001$ ). Comparisons are made to the positive endogenous control provided by the manufacturer cytokines, and receptors and 41 growth factors impregnated in small circles on membranes along with positive and negative controls. All antibodies are bound in small duplicate circles on the membranes. Drawn circles indicate cytokines that were enhanced above the background. Unstimulated PBMC produce small amounts of cytokines spontaneously thereby preventing their use as controls

that soluble $\mathrm{CI}$ was used rather than plate-bound $\mathrm{CI}$ in the PBMC culture favors the concept that $\mathrm{CI}$ functions as an antigen, which triggers the production of cytokines which cause SSc fibroblasts to assume a phenotype of impaired MMP-1 production.

The pathogenesis of SSc is marked by the overabundance of collagen and skin fibrosis where collagen fibers accumulate in the dermis. Uncontrolled production of collagen and other extracellular matrix proteins produced by fibroblasts contributes to the fibrosis [20]. MMP-1 is the major proteinase involved in the degradation of CI and CIII. Although decreases in MMP-1 in fibroblasts from patients with early SSc have been reported [21], the results have been controversial [22]. Our data support the concept that an important component of the pathogenesis of SSc is the diminished capacity for production of MMP-1 by fibroblasts. Our data are unique in indicating that the reduction of fibroblast MMP-1 is mediated by cytokines derived from PBMC stimulated by $\mathrm{CI}$. It is possible that $\mathrm{CI}$ is functioning as an autoantigen or a co-stimulator of $\mathrm{T}$ cells in eliciting these cytokines. 

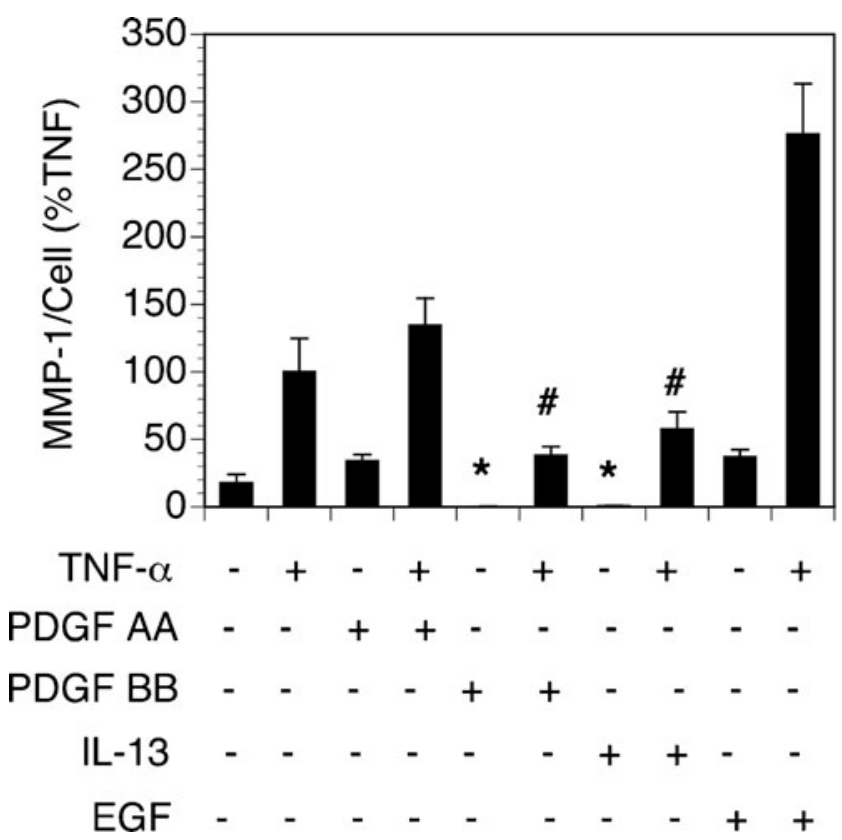

Fig. 4 SSc dermal fibroblasts cultured for 14 days with proinflammatory cytokines followed by stimulation with TNF- $\alpha$ for $24 \mathrm{~h}$. Inhibition of constitutively produced MMP-1 as well as TNF$\alpha$-stimulated MMP-1 production was observed when SSc 008 fibroblasts were cultured with PDGF-BB and IL-13 when compared to SSc fibroblasts cultured with the medium alone for 14 days \pm TNF- $\alpha$. MMP-1 was increased when fibroblasts were cultured for 14 days with PDGF-AA or EGF and then stimulated $24 \mathrm{~h}$ with TNF- $\alpha .{ }^{*} P<0.05$ when compared to supernatants from fibroblasts cultured with the complete medium alone (first bar on the left). ${ }^{\#} P<0.05$ when compared to supernatants from fibroblasts cultured with the complete medium plus TNF (second bar from the left)

The expression of IL-13 and its receptor complex is increased in SSc skin samples, correlating with modified Rodnan skin scores (Aliprantis; ACR 2010) and with nail fold capillary patterns [23-25]. Our studies demonstrate that pooled supernatants from adult SSc PBMC co-cultured with CI contain significant levels of many profibrotic cytokines compared to controls (PDGF-AA, PDGF-BB, PDGF-AB, EGF, IGFBP-2, HGF, MCSFR, and IL-13) [6, 26-29]. Our studies of pediatric patients with SSc have shown that CI induces increased expression of PDGF-AA, PDGF-BB, EGF TNF- $\alpha$, and IL-13. The overlap of cytokines found in juvenile and adult SSc compared to that seen in localized disease may in fact be reflective of severity of skin and organ involvement between these two diseases. Moreover, the functional importance of these cytokines is demonstrated by our results that prolonged incubation of PDGF-BB and IL-13 significantly inhibited TNF- $\alpha$, induced MMP-1 production by SSc dermal fibroblasts, and highlight the importance of these cytokines in dcSSc. Together, the interaction of PBMC with $\mathrm{CI}$ and the elicited cytokines stimulates a pathway inherent in SSc fibroblasts that inhibits MMP-1 expression and contributes to the fibrosis in this disease.
In the present study, we have shown that cytokines produced by CI-stimulated PBMC from patients with dcSSc suppress MMP-1 production. These data suggest a role for activation of lymphocytes and/or monocytes in a subgroup of patients with dcSSc and suggest a cytokine-mediated pathway involved in the pathogenesis of the extensive fibrosis seen in these patients. Since we have shown that exposure to oral CI may benefit late-phase dcSSc, our new results have important implications for future treatments for SSc [30].

\section{Patients and methods}

Patient population This study was conducted using protocols approved by the Institutional Review Board of participating institutions. The Declaration of Helsinki protocols were followed; patients gave their written informed consent. Juvenile patients with dcSSc (ages 16 years or less) were included in this study who met the PREs/ACR/EULAR classification of juvenile systemic sclerosis [31]. Adult patients (aged 18-70) met the American College of Rheumatology preliminary criteria for diagnosis of SSc. Patients with LS met the classification criteria for morphea or linear scleroderma [2, 32].

Preparation of bovine native CI and $\alpha 1$ (I) chains Native CI was isolated and purified from fetal bovine skin as previously described [33]. Homogeneity of CI and $\alpha 1$ (I) was confirmed using SDS-PAGE and by cyanogen bromide peptide mapping. The purified CI was dissolved in cold $0.1 \mathrm{M}$ acetic acid at $4 \mathrm{mg} / \mathrm{ml}$ and stored frozen at $-70^{\circ} \mathrm{C}$. $\alpha 1$ (I) was isolated from denatured bovine CI as previously described [34]. Purified $\alpha 1$ (I) was lyophilized and stored at $-20^{\circ} \mathrm{C}$ in a desiccator.

Specific reagents Human recombinant TNF- $\alpha$, IL-13, PDGF-AA, PDGF-BB, and EGF were purchased from R\&D systems (Minneapolis, MN). Anti-rabbit IgG was purchased from GE Healthcare (UK). The antibody to human MMP-1 (rabbit) was made to a latent and truncated peptide, expressed in Escherichia coli, and purified in the laboratory. MMP-1 was detected by ELISA (R\&D systems) and cytokines by RayBio Human Cytokine Antibody Arrays (RayBiotech, Inc.).

Production of PBMC culture supernatants Blood samples were collected in EGTA-containing glass tubes and the PBMC isolated over Histopaque (Sigma Chemical Co., St. Louis, MO) cushions by isopynic centrifugation. The PBMC were plated at $2 \times 10^{6}$ cells per well, cultured in Dulbeccos's high-glucose modified eagle medium (DMEM) containing 10\% fetal bovine serum (FBS), penicillin 
(100 units/ml), streptomycin $(100 \mathrm{ug} / \mathrm{ml})$, and $100 \times$ Glutamax (Invitrogen Corp.) [19] (hereafter called "Complete DMEM"). Cells were cultured at $37^{\circ} \mathrm{C}$ with $5 \% \mathrm{CO}_{2}$ for 6 days with and without purified bovine collagen $\alpha 1(\mathrm{I})$ $(25 \mu \mathrm{g} / \mathrm{ml})$ after which the supernatants were collected, pooled, and stored in $-80^{\circ} \mathrm{C}$ until use. In one experiment, PBMC from ten adult patients with dcSSc were collected and cultured for 6 days with native bovine CI $(10 \mu \mathrm{g} / \mathrm{ml})$, prior to pooling and storing at $-70^{\circ} \mathrm{C}$.

Dermal fibroblast cultures Four normal and four SSc fibroblast lines (two from biopsies of lesional and two from nonlesional skin of dsSSc patients) were grown for 2-3 weeks in eagle minimum essential medium, with $9 \%$ fetal calf serum (FCS), $100 \mu / \mathrm{ml}$ penicillin, $100 \mu \mathrm{g} / \mathrm{ml}$ streptomycin, and amphotericin B $(1 \mu \mathrm{g} / \mathrm{ml})$ hereafter referred to as "complete medium." Thirty percent (by volume) of supernatant from a culture of CI-stimulated dcSSc PBMC (from a pool of ten adult patients with dcSSc, sterile filtered and aliquoted into individual vials) or $30 \%$ medium (by volume) alone or plus native CI $(10 \mu \mathrm{g} / \mathrm{ml})$ of high-glucose DMEM containing the same lot of FBS used in the SSc PBMC cultures was added to each plate containing each cell line. The medium in the cultures was changed every 7 days, and a new SSc PBMC culture supernatant or medium control (each $30 \%$ by volume) was added at each refeeding. Cells were then trypsinized and passed into new $100-\mathrm{mm}$ tissue culture plates and cultured for 3 days in the complete medium. Fibroblasts were then trypsinized and transferred to Costar tissue culture plates at a density of $10^{5}$ cells/well and grown for 3 days in the complete medium. The medium was then changed to the complete medium containing 5\% FBS for $24 \mathrm{~h}$, changed again to a fresh complete medium with $5 \%$ FCS containing either $5 \mathrm{ng} / \mathrm{ml}$ human recombinant TNF- $\alpha$ (R and D Systems, Minneapolis, MN) in PBS containing $0.1 \%$ bovine serum albumin (BSA, Sigma) or PBS with $0.1 \% \mathrm{BSA}$ as a control. After $48 \mathrm{~h}$, culture supernatants were harvested. Culture supernatants were assayed by ELISA or Western blot analysis to quantitate MMP-1 protein as previously described [35].

Culture of SSc fibroblasts with supernatants from PBMC cultures Fibroblasts were grown from dermal biopsies from similar locations on the forearm of normal volunteers or patients with dcSSc. SSc fibroblast cell line 008 (SSc 008) passages $5-15$, that had regained the ability to synthesize MMP-1 upon stimulation with TNF- $\alpha$, was also used for comparing the effect of the supernatant from the culture of PBMC from different donors. This SSc fibroblast line, grown from the fibrotic skin on the volar forearm of a patient with dcSSc, was resistant to TNF- $\alpha$ stimulation of MMP-1 synthesis during the first few subpassages but became more responsive to TNF- $\alpha$ after passage. The SSc 008 fibroblasts were plated at $5 \times 10^{4}$ cells/well in Costar 24 well and grown in high-glucose DMEM containing $10 \%$ FCS. Supernatants from 6-day PBMC cultures were removed from $-80^{\circ} \mathrm{C}$ storage, allowed to thaw at $37^{\circ} \mathrm{C}$, and then centrifuged at 190 relative centrifugal force for $10 \mathrm{~min}$. Additions to fibroblast cultures consisted of the following: collagen alone in complete DMEM (complete DMEM incubated 6 days with $12.5 \mu \mathrm{l}(12.5 \mu \mathrm{g})$ of $\alpha \mathrm{l}(\mathrm{I})$ without PBMC in wells), PBMC supernatant (PBMC; DMEM incubated 6 days with PBMC from scleroderma patients), and collagen supernatant (+CI/PBMC; DMEM incubated for 6 days with PBMC and $12.5 \mu \mathrm{l}$ of $12.5 \mu \mathrm{g} \alpha 1(\mathrm{I}))$. The PBMC supernatants or complete medium was added at $30 \% v / v$ to triplicate well cultures of SSc 08 fibroblasts. The medium was changed every 3 days for a total of 21 days, then DMEM supplemented with 5\% FBS was placed on the fibroblasts for $24 \mathrm{~h}$ prior to adding TNF- $\alpha 5 \mathrm{ng} / \mathrm{ml}$ for an additional $24 \mathrm{~h}$. Fibroblast cultures were then collected and stored at $4^{\circ} \mathrm{C}$ until analysis by Western blot or ELISA to quantitate MMP-1 level. Fibroblasts were harvested for cell number quantification using CyQuant (Invitrogen, Eugene, OR).

Analysis of MMP-1 Fibroblast culture supernatants were analyzed for MMP-1 protein by Western blot analysis or by ELISA. Supernatants were resolved on 9\% SDS-PAGE and electrotransferred to a PVDF membrane, blocked in 5\% powdered milk in TBS ( $1 \mathrm{M}$ Tris, $\mathrm{pH} 7.4 ; 4 \mathrm{M} \mathrm{NaCl}$; deionized water) for $1 \mathrm{~h}$, then soaked in primary MMP-1 antibody. The membrane was washed and incubated with anti-MMP-1 antibody, and then, alkaline phosphataseconjugated anti-rabbit IgG was used to visualize immunoreactive bands with ECF substrate. Densities were normalized to cell proliferation values (number of cells) and calculated as a percent of the TNF- $\alpha$ control value (Storm 860 Molecular Dynamics, Sunnyvale, CA, and Typhoon GE Healthcare Biosciences $\mathrm{AB}$, Sweden) and analyzed using ImageQuant TL 7.0 (GE Healthcare, Sweden). In some experiments, human total MMP-1 Duoset (R\&D) was used to quantify MMP-1.

Cytokine analysis Cytokine analysis was performed on 6-day complete DMEM and $+\mathrm{CI} / \mathrm{PBMC}$ pooled supernatants obtained from 6-day culture of PBMC from ten dcSSc patients cultured with $10 \mu \mathrm{g} / \mathrm{ml}$ native CI using RayBio Human Cytokine Antibody Arrays VI, 6.1, and VI, 7.1 (RayBiotech, Inc.).

Further analysis was done on CI-stimulated 6-day PBMC supernatants from two juvenile LS patients and one dsSSc juvenile patient. For this analysis, membrane arrays were custom made for by the manufacturer for detection of PDGF-AA, PDGF-BB, IL-13, TNF- $\alpha$, EGF, MCSCR, insulin-like binding protein 2 (IGFBP-2), IL-1 $\beta$, HGF, and 
IL-17. These cytokines were selected based on the preliminary cytokine data obtained from pooled supernatants from dcSSc patients. All procedures for this part of the experiment were followed according to the manufacturer's instructions. Each blot of cytokine array membranes was normalized to irrelevant endogenous controls included on the blot by the manufacturer to allow comparisons between blots and analyzed using Typhoon chemiluminescence.

To study the effect of long-term exposure of fibroblasts to cytokines/growth factors, fibroblasts were plated in 100-mm tissue culture plates at $4 \times 10^{5}$ cells per plate and then cultured with human recombinant PDGF-AA, PDGF-BB, IL-13, and EGF for a total of 14 days. The medium was changed every $72 \mathrm{~h}$ with addition of fresh medium containing the specific cytokine/growth factor. Fibroblasts were passed by trysinization and then plated with the complete medium containing $10 \%$ FCS in Costar 24-well tissue culture plates at $5 \times 10^{4}$ per well. These fibroblasts were then grown in the complete medium for 1 week with medium change every $72 \mathrm{~h}$. Fibroblasts were then grown in DMEM containing 5\% FBS for $72 \mathrm{~h}$, followed by $24 \mathrm{~h}$ with TNF- $\alpha$ stimulation. Human total MMP-1 Duoset was used to determine MMP-1.

Statistical analysis An all-or-none comparison was made between groups. ANOVA and Fisher's exact test were used for statistical analysis due to a small sample size. $P<0.05$ was considered significant. Data are reported with bars indicating the standard deviation of the mean.

Acknowledgments Dr. Brown is the recipient of an Arthritis Foundation Postdoctoral Fellowship Award. This work was also supported by USPHS grants AR-55661, AR-55266, AR-007317, and programdirected funds from the U.S. Department of Veterans Affairs (AEP and $\mathrm{KAH}$ ) and the LeBonheur Children's Research Hospital. SSc fibroblast lines 1685 and 1822 were obtained from the University of Texas Health Science Center at Houston SSc Cell Culture Core.

\section{Disclosures None.}

Open Access This article is distributed under the terms of the Creative Commons Attribution License which permits any use, distribution, and reproduction in any medium, provided the original author(s) and the source are credited.

\section{References}

1. Atamas SP, Luzina IG, Ingels J et al (2010) Stimulation with type I collagen induces changes in gene expression in peripheral blood mononuclear cells from patients with diffuse cutaneous systemic sclerosis (scleroderma). Clin Exp Immunol 161:426-435

2. Peterson LS, Nelson AM, Su WP (1995) Classification of morphea (localized scleroderma). Mayo Clin Proc 70:1068-1076

3. Johnson SR, Laxer RM (2006) Classification in systemic sclerosis. J Rheumatol 33:840-841
4. Fett N, Werth VP (2011) Update on morphea: part I. Epidemiology, clinical presentation, and pathogenesis. J Am Acad Dermatol 64:217-228, quiz 29-30

5. Gu YS, Kong J, Cheema GS, Keen CL, Wick G, Gershwin ME (2008) The immunobiology of systemic sclerosis. Semin Arthritis Rheum 38:132-160

6. Yamane K, Ihn H, Tamaki K (2003) Epidermal growth factor upregulates expression of transforming growth factor beta receptor type II in human dermal fibroblasts by phosphoinositide 3-kinase/ Akt signaling pathway: resistance to epidermal growth factor stimulation in scleroderma fibroblasts. Arthritis Rheum 48:1652-1666

7. Yuan W, Varga J (2001) Transforming growth factor-beta repression of matrix metalloproteinase-1 in dermal fibroblasts involves smad3. J Biol Chem 276:38502-38510

8. Distler JH, Allanore Y, Avouac J et al (2009) EULAR Scleroderma Trials and Research group statement and recommendations on endothelial precursor cells. Ann Rheum Dis 68:163-168

9. Kantor TV, Friberg D, Medsger TA Jr, Buckingham RB, Whiteside TL (1992) Cytokine production and serum levels in systemic sclerosis. Clin Immunol Immunopathol 65:278-285

10. Koch AE, Kronfeld-Harrington LB, Szekanecz Z et al (1993) In situ expression of cytokines and cellular adhesion molecules in the skin of patients with systemic sclerosis. Their role in early and late disease. Pathobiol: J Immunopathol, Mol Cell Biol 61:239-246

11. Fleischmajer R, Perlish JS, West WP (1977) Ultrastructure of cutaneous cellular infiltrates in scleroderma. Arch Dermatol 113:1661-1666

12. Roumm AD, Whiteside TL, Medsger TA Jr, Rodnan GP (1984) Lymphocytes in the skin of patients with progressive systemic sclerosis. Quantification, subtyping, and clinical correlations. Arthritis Rheum 27:645-653

13. Fritz J, Sandhofer M (1977) Cellular immune phenomenon in scleroderma. Dermatologica 154:129-137

14. Gurram M, Pahwa S, Frieri M (1994) Augmented interleukin-6 secretion in collagen-stimulated peripheral blood mononuclear cells from patients with systemic sclerosis. Ann Allergy 73:493496

15. Hawrylko E, Spertus A, Mele CA, Oster N, Frieri M (1991) Increased interleukin-2 production in response to human type I collagen stimulation in patients with systemic sclerosis. Arthritis Rheum 34:580-587

16. Mackel AM, DeLustro F, DeLustro B, Fudenberg HH, LeRoy EC (1982) Immune response to connective tissue components of the basement membrane. Connect Tissue Res 10:333-343

17. McKown KM, Carbone LD, Bustillo J, Seyer JM, Kang AH, Postlethwaite AE (2000) Induction of immune tolerance to human type I collagen in patients with systemic sclerosis by oral administration of bovine type I collagen. Arthritis Rheum 43:1054-1061

18. Stuart JM, Postlethwaite AE, Kang AH (1976) Evidence for cellmediated immunity to collagen in progressive systemic sclerosis. $\mathrm{J}$ Lab Clin Med 88:601-607

19. Warrington KJ, Nair U, Carbone LD, Kang AH, Postlethwaite AE (2006) Characterisation of the immune response to type I collagen in scleroderma. Arthritis Res Ther 8:R136

20. Varga JA, Trojanowska M (2008) Fibrosis in systemic sclerosis. Rheum Dis Clin North Am 34:115-143; vii

21. Kuroda K, Shinkai H (1997) Differential regulations of matrix metalloproteinases and tissue inhibitors of metalloproteinases in dermal fibroblasts by dibutyril cyclic AMP. J Dermatol Sci 15:95100

22. Becvar R, Hulejova H, Braun M, Stork J (2007) Collagen degradation products and proinflammatory cytokines in systemic and localized scleroderma. Folia Biol (Praha) 53:66-68

23. Riccieri V, Rinaldi T, Spadaro A et al (2003) Interleukin-13 in systemic sclerosis: relationship to nailfold capillaroscopy abnormalities. Clin Rheumatol 22:102-106 
24. Gourh P, Arnett FC, Assassi S et al (2009) Plasma cytokine profiles in systemic sclerosis: associations with autoantibody subsets and clinical manifestations. Arthritis Res Ther 11:R147

25. Hasegawa M, Fujimoto M, Kikuchi K, Takehara K (1997) Elevated serum levels of interleukin 4 (IL-4), IL-10, and IL-13 in patients with systemic sclerosis. J Rheumatol 24:328-332

26. Hasegawa M, Fujimoto M, Kikuchi K, Takehara K (1997) Elevated serum tumor necrosis factor-alpha levels in patients with systemic sclerosis: association with pulmonary fibrosis. J Rheumatol 24:663-665

27. Hasegawa M, Sato S, Nagaoka T, Fujimoto M, Takehara K (2003) Serum levels of tumor necrosis factor and interleukin-13 are elevated in patients with localized scleroderma. Dermatology 207:141-147

28. Fujii H, Hasegawa M, Takehara K, Mukaida N, Sato S (2002) Abnormal expression of intracellular cytokines and chemokine receptors in peripheral blood $\mathrm{T}$ lymphocytes from patients with systemic sclerosis. Clin Exp Immunol 130:548-556

29. Restrepo JF, Guzman R, Rodriguez G, Iglesias A (2003) Expression of transforming growth factor-beta and platelet-derived growth factor in linear scleroderma. Biomedica 23:408-415
30. Postlethwaite AE, Wong WK, Clements P et al (2008) A multicenter, randomized, double-blind, placebo-controlled trial of oral type I collagen treatment in patients with diffuse cutaneous systemic sclerosis: I. Oral type I collagen does not improve skin in all patients, but may improve skin in late-phase disease. Arthritis Rheum 58:1810-1822

31. Zulian F, Woo P, Athreya BH et al (2007) The Pediatric Rheumatology European Society/American College of Rheumatology/ European League against rheumatism provisional classification criteria for juvenile systemic sclerosis. Arthritis Rheum 57:203-212

32. Laxer RM, Zulian F (2006) Localized scleroderma. Curr Opin Rheumatol 18:606-613

33. Kang AH, Piez KA, Gross J (1969) Characterization of the alphachains of chick skin collagen and the nature of the NH2-terminal cross-link region. Biochemistry 8:3648-3655

34. Kang AH, Igarashi S, Gross J (1969) Characterization of the cyanogen bromide peptides from the alpha- 2 chain of chick skin collagen. Biochemistry 8:3200-3204

35. Staatz WD, Walsh JJ, Pexton T, Santoro SA (1990) The alpha 2 beta 1 integrin cell surface collagen receptor binds to the alpha 1 (i)-cb3 peptide of collagen. J Biol Chem 265:4778-4781 